\title{
M-Learning: uma revisão sistemática dos desafios no desenvolvimento de tecnologias educacionais para dispositivos digitais móveis
}

\author{
Ewerton Carlos de Araujo Assis ${ }^{1}$ \\ ${ }^{1}$ Universidade Federal do ABC (UFABC) - Santo André - SP - Brazil \\ carlos.assiseufabc.edu.br
}

\begin{abstract}
Resumo. A presente revisão sistemática da literatura analisa trabalhos publicados entre 2014 e 2018 a fim de evidenciar os principais desafios ao conceber, desenvolver e implantar tecnologias educacionais mediadas por dispositivos digitais móveis. Sobretudo, são analisadas as características que a realidade brasileira impõe nesta área. Os protocolos para esta pesquisa são organizados em questões-chaves que nortearam a escolha e a avaliação dos artigos $e$ sua relevância. Ao final, são discutidos os principais desafios levantados pela análise dos trabalhos: a adaptação de objetos de aprendizagem e suas interfaces; interatividade, mobilidade e disponibilidade de conexão à Internet; design universal e participativo; realidade virtual ou aumentada e experiências locativas através de dispositivos digitais móveis; e localização e internacionalização de conteúdos.
\end{abstract}

\begin{abstract}
The present work aims at a systematic literature review of the main challenges addressed in recent works, from 2014 to 2018, focused on educational technologies mediated by mobile digital devices, in order to obtain an overview of how those challenges have been addressed or whether they are still open for possible developments. It focuses, above all, on the characteristics and challenges that the Brazilian reality presents in this field. A discussion of this systematic review, its methodology and its possible biases and conclusions are also presented. The main challenges are related to the localization and internationalization of content; the adaptation of learning objects and their interfaces; interactivity; mobility and availability of Internet connection; universal and participatory design (co-design); virtual or augmented reality; and locative experiences through mobile digital devices.
\end{abstract}

\section{Introdução e motivação}

Dispositivos digitais móveis, em seus diversos formatos e configurações, estão cada vez mais ubíquos, ampliando cada vez mais o alcance de tecnologias digitais a diversos usuários. No Brasil, com base em dados levantados pelo Instituto Brasileiro de Geografia e Estatística (IBGE) [IBGE 2018], 64,7\% da população brasileira com 10 anos de idade ou mais tem acesso à Internet; destes, 94,6\% preferem o uso de dispositivos móveis como meio preferido para acesso à Internet. Em relatório desenvolvido e divulgado pela GSMA (Global System for Mobile Communications), 75\% da população brasileira é formada por usuários únicos de telefonia móvel [GSMA 2018]. Ainda com base no mesmo relatório, o Brasil se consolida como o quinto maior mercado de smartphones, 
VIII Congresso Brasileiro de Informática na Educação (CBIE 2019)

Anais do XXV Workshop de Informática na Escola (WIE 2019)

com 200 milhões de dispositivos ativos. A partir desta realidade, existe uma crescente necessidade de adaptar, conceber e implantar aplicações para dispositivos digitais móveis [Dehlinger and Dixon 2011], respeitando seus desafios e características próprias.

O uso de tecnologia digital como meio para apresentar e desenvolver conteúdos educacionais, no entanto, não é uma novidade. O e-Learning é discutido desde a origem do computador digital somada à sua popularização alavancada pela emergência da Internet, estabelecendo-se parâmetros para como melhor conceber, desenvolver e mediar conteúdos educacionais através destes meios ou multimeios [Fernandes et al. 2012]. Assim como a convergência de conteúdos não-digitais para computadorizados apresentaram (e ainda apresentam) desafios [Fernandes et al. 2012], é importante entender a realidade que a convergência de dispositivos digitais "tradicionais" (computadores desktop, notebooks etc.) para dispositivos móveis apresenta: seus próprios dilemas e desafios.

A mediação de tecnologias e conteúdos educacionais através de dispositivos digitais móveis é frequentemente referenciada e estudada sob os termos mobile learning ou m-Learning [Martín and Carro 2009] [Dochev and Hristov 2006].

Dado este contexto de universalização do acesso a conteúdos educacionais através de dispositivos móveis, o presente trabalho tem por finalidade realizar uma revisão sistemática da literatura dos principais desafios abordados em trabalhos focados em conteúdos educacionais mediados por dispositivos digitais móveis, a fim de obter-se um panorama de como são solucionados ou se ainda se encontram em aberto para possíveis desenvolvimentos. A intenção, a partir desta revisão, é encontrar lacunas que poderão ser desenvolvidas e discutidas através de um arcabouço para a construção de software educacional a ser implantado em dispositivo digital móvel, tendo por base as características da realidade brasileira.

O texto está organizado da seguinte forma: na seção 2, discute-se a metodologia de trabalho, como a revisão bibliográfica foi feita e quais os principais vieses que emergem a partir dos parâmetros desta revisão sistemática da literatura; na seção 3, discute-se os principais desafios na convergência de conteúdos educacionais digitais para a realidade móvel; e, por fim, a seção 4 apresenta as conclusões que podem ser obtidas a partir do presente trabalho e futuros desenvolvimentos.

\section{Metodologia e questionamentos-chave}

A presente revisão sistemática da literatura foi realizada a partir das seguintes bases de trabalhos científicos: artigos indexados pelo Google Scholar (GS); artigos apresentados e/ou publicados no Simpósio Brasileiro de Informática na Educação (SBIE); e artigos apresentados e/ou publicados nos Anais dos Workshops do Congresso Brasileiro de Informática na Educação (CBIE). Respectivamente, estas bases de pesquisa proveram 106.000, 85 e 58 trabalhos totais, a partir das chaves de busca: "dispositivo móvel" OR "dispositivos móveis", no caso do SBIE e CBIE, e "mobile learning", no casso do GS.

Dada a totalidade de artigos encontrados, os seguintes critérios foram adotados para selecionar os trabalhos para a revisão sistemática: (1) artigos publicados/apresentados entre 2014 e 2018 (inclusivo); (2) artigos encontrados pelo indexador GS que tivessem 40 ou mais citações por outros trabalhos; e (3) apenas artigos que apresentavam ou discutiam os desafios na implementação de tecnologias ou soluções educacionais baseadas em tecnologia digital móvel (smartphones ou tablets). Ao final desta 
VIII Congresso Brasileiro de Informática na Educação (CBIE 2019)

Anais do XXV Workshop de Informática na Escola (WIE 2019)

filtragem, foram selecionados 11, 30 e 30 artigos das bases GS, SBIE e CBIE, respectivamente.

Estas decisões metodológicas para a revisão sistemática poderão, eventualmente, ter excluído trabalhos que apresentem insumos relevantes para a presente revisão sistemática e a futura construção de um arcabouço de software para desenvolvimento de soluções educacionais. No entanto, a coleção de trabalhos selecionados apresenta uma análise relevante do que tem sido desenvolvido na área e nos possíveis desafios em aberto, sobretudo após o advento e a massificação dos dispositivos digitais móveis denominados smartphones.

\subsection{Questionamentos-chave e análise dos trabalhos de referência para a revisão sistemática}

Ao analisar as proposições e soluções apresentadas pelos trabalhos selecionados para a revisão sistemática, os seguintes questionamentos-chave foram utilizados para estabelecer a relevância dos trabalhos frente aos propósitos da revisão, a fim de obter-se respostas que identificassem os principais desafios: (1) o trabalho apresenta uma solução original ou apresenta uma solução (ou soluções) de terceiro(s)?; (2) é feita uma apresentação dos objetivos esperados ao se desenvolver essa solução (ou soluções) e quais são os critérios de avaliação da satisfação destes objetivos previamente estabelecidos?; (3) a solução (ou soluções) utiliza componentes de software que evoluíram ao longo do tempo ou que ainda estão disponíveis?; e (4) o trabalho sustenta-se, dentre outros conceitos, na proposta de $m$ Learning ou utiliza um dispositivo computacional móvel apenas como uma possibilidade de sistema para implantação da solução, sem pouco explorar os porquês desta decisão?

\section{Principais desafios}

Desde a emergência de dispositivos digitais móveis é discutido quais são os principais desafios na concepção, arquitetura, projeto, desenvolvimento e avaliação de soluções em software para estas configurações [Dehlinger and Dixon 2011]. Soma-se a estes fatores características específicas a soluções educacionais [Fernandes et al. 2012]. Neste sentido, compreender como estes desafios emergem e como podem ser solucionados provê um aumento na qualidade da solução desenvolvida e na maturidade dos processos envolvidos na construção deste software.

A partir da coleção de trabalhos selecionados para esta revisão sistemática, e tendo como referência para análise os questionamentos-chave apresentados na seção 2, os principais desafios abordados ao conceber e desenvolver soluções baseadas em m-Learning são: (1) a adaptação de conteúdos e interfaces (UI); interatividade e experiência do usuário (UX); mobilidade e disponibilidade de conexão à Internet; e design universal e design participativo - os quais correspondem a $38 \%$ dos trabalhos analisados. Sobretudo, estes desafios relacionam-se a como prover soluções que atendam as necessidades e realidades dos usuários e as características próprias dos dispositivos móveis e demais recursos externos (como conexão à Internet); (2) realidade virtual (VR) ou aumentada (AR) e experiências locativas através de dispositivos digitais móveis — os quais correspondem a $21 \%$ dos trabalhos avaliados. A partir dos dispositivos digitais móveis e com a convergência destes com outras tecnologias, como VR e AR, novos desafios surgiram em como construir soluções que façam bom 
VIII Congresso Brasileiro de Informática na Educação (CBIE 2019)

Anais do XXV Workshop de Informática na Escola (WIE 2019)

uso destas possibilidades de interação [Biedert et al. 2010] [Modesto and Mustaro 2014]; e (3) a localização (110n) e a internacionalização (i18n) de conteúdos, que corresponde a $3 \%$ dos trabalhos selecionados. Um dos aspectos relevantes quanto à utilização de softwares em sala de aula é a possibilidade de adaptar, através da internacionalização e da localização, objetos de aprendizagem que foram desenvolvidos em contexto nacional ou cultural distinto. Desafios emergem ao tentar adaptar as bases curriculares de ensino, os níveis de ensino, idade dos discentes, abordagens pedagógicas, preparação e capacidade dos docentes em utilizar estas tecnologias etc. [da Silva and da Graça Moreira da Silva 2014] [O'Bannon and Thomas 2014] [do Nascimento and de Castro Filho 2015] [Ferrete and da Conceição Andrade 2017].

Além destes desafios principais, outros também foram identificados, ainda que não sejam exclusivamente pertinentes ao m-Learning. Os seguintes desafios de projeto foram obtidos a partir da revisão sistemática, em menor frequência frente aos principais: (1) multimodalidade, ou a possibilidade de utilizar um objeto de aprendizagem através de diversos dispositivos digitais, como celulares smartphone, TV (digital), notebooks etc.; (2) a união dos conceitos de $e$-Learning com m-Learning e u-Learning - este sendo compreendido como o ensino ubíquo [Cope and Kalantzis 2009]; (3) a disponibilidade dos objetos de aprendizagem em repositórios que fossem em si mesmo móveis (que dispusessem de interfaces que poderiam ser utilizadas em dispositivos móveis) ou que fossem específicos para objetos de aprendizagem móveis (OAM); (4) objetos de aprendizagem que estivessem sob a análise de sistemas baseados em learning analytics; (5) a gamificação de objetos de aprendizagem com a finalidade de aprofundar o engajamento dos alunos (e usuários em geral) com a solução tecnológica; e (6) a integração programática de múltiplos sistemas de software em ambientes de m-Learning, possibilitando que diversos serviços sejam integrados, como learning analytics, ambientes virtuais de aprendizado (AVA), sugestão de conteúdo etc.

\section{Conclusões e trabalhos futuros}

Dispositivos móveis estão cada vez mais presentes na vida dos usuários de tecnologias digitais, sendo uma solução de alcance e horizontalidade para o acesso e divulgação de conteúdos educacionais digitais. Conhecer quais são os principais desafios na concepção, adaptação, implementação e implantação de conteúdos e tecnologias educacionais mediadas por tecnologia digital em dispositivos móveis torna-se importante para potencializar o acesso à educação mediada por tecnologias digitais, além de subsidiar a concepção de arcabouços e padrões de projetos para a construção de soluções neste escopo de implantação. Os principais desafios abordados não apresentaram arcabouços ou propostas de soluções arquiteturais que possam ser aplicadas em mais de um contexto de solução, justificando uma possível e positiva intervenção nesta área.

A presente revisão sistemática, a partir de sua metodologia de pesquisa e revisão dos trabalhos encontrados e a partir dos questionamentos-chave levantados, apresenta quais foram os desafios mais preponderantes ao desenvolver soluções baseadas em $e$ Learning e m-Learning. As contribuições obtidas possibilitarão delinear parâmetros para conceber um arcabouço com padrões de projetos para a construção de tecnologia educacional a ser implantada em dispositivos digitais móveis, sobretudo nos desafios relacionados a interatividade e experiência de usuário, mobilidade, design universal e participativo, realidade virtual ou aumentada e localização e internacionalização de conteúdos. 
VIII Congresso Brasileiro de Informática na Educação (CBIE 2019)

Anais do XXV Workshop de Informática na Escola (WIE 2019)

\section{Referências}

Biedert, R., Buscher, G., Schwarz, S., Hees, J., and Dengel, A. (2010). Text 2.0. In CHI '10 Extended Abstracts on Human Factors in Computing Systems, pages 4003-4008.

Cope, B. and Kalantzis, M. (2009). Ubiquitous learning. University of Illinois Press, 1st edition.

da Silva, J. M. and da Graça Moreira da Silva, M. (2014). A Escola e o Currículo em Tempos de Mobilidade e Conexão: o uso dos computadores portáteis na educação. In Workshops do 3 o Congresso Brasileiro de Informática na Educação (CBIE 2014).

Dehlinger, J. and Dixon, J. (2011). Mobile Application Software Engineering: Challenges and Research Directions.

do Nascimento, K. A. S. and de Castro Filho, J. A. (2015). Aprendizagem móvel e suas tecnologias: uma revisão sistemática da literatura. In Anais dos Workshops do IV Congresso Brasileiro de Informática na Educação (CBIE 2015), pages 721-730.

Dochev, D. and Hristov, I. (2006). Mobile Learning Applications: Ubiquitous Characteristics and Technological Solutions. Cybernetics and Information Technologies, 6(3):63-74.

Fernandes, K. T., da Trindade, G. O., Rêgo, A. H. G., de Miranda, L. C., Lucena, M. J. N. R., and Gomes, A. V. (2012). e-Learning via Dispositivos Móveis no Brasil: Estado da Arte e Desafios à Luz do Acesso Participativo e Universal do Cidadão Brasileiro ao Conhecimento. In Workshop de Desafios da Computação Aplicada à Educação.

Ferrete, A. A. S. S. and da Conceição Andrade, C. (2017). Formação docente: percepções dos professores sobre o uso das tecnologias móveis digitais no processo de ensino e aprendizagem. In Anais dos Workshops do VI Congresso Brasileiro de Informática na Educação (WCBIE 2017), pages 515-523.

GSMA (2018). The Mobile Economy 2018. Technical report, GSMA. Último acesso em 22 de agosto de 2018; disponível em: https://wWw.gsma.com/ mobileeconomy/.

IBGE (2018). PNAD Contínua TIC 2016: 94,2\% das pessoas que utilizaram a Internet o fizeram para trocar mensagens. Último acesso em 20 de agosto de 2018; disponível em: https: //cod.ibge.gov.br/3EAJV.

Martín, E. and Carro, R. M. (2009). Supporting the Development of Mobile Adaptive Learning Environments: A Case Study. IEEE Transactions on Learning Technologies, 2(1):23-36.

Modesto, F. A. C. and Mustaro, P. N. (2014). Revisão Sistemática para estudo de Interação Criança-Computador associada a RA e Jogos Digitais. In Workshops do 3 o Congresso Brasileiro de Informática na Educação (CBIE 2014), pages 554-563.

O'Bannon, B. W. and Thomas, K. (2014). Teacher perceptions of using mobile phones in the classroom: Age matters! Computers \& Education, 74:15-25. 